Published in final edited form as:

J Psychosom Res. 2020 April ; 131: 109793. doi:10.1016/j.jpsychores.2019.109793.

\title{
Perceived Weight Discrimination and Performance in Five Domains of Cognitive Function
}

\author{
Angelina R. Sutin ${ }^{1,}$, Yannick Stephan ${ }^{2}$, Mary A. Gerend ${ }^{1}$, Eric Robinson ${ }^{3}$, Michael Daly ${ }^{4}$, \\ Antonio Terracciano ${ }^{1}$ \\ ${ }^{1}$ Florida State University College of Medicine \\ ${ }^{2}$ Euromov, University of Montpellier \\ ${ }^{3}$ University of Liverpool \\ ${ }^{4}$ Maynooth University
}

\section{Abstract}

Objective-Individuals who perceive unfair treatment because of their body weight have been found to be at increased risk of poor health outcomes, including risk of dementia. The present research examines the relation between weight discrimination and performance in five cognitive domains (episodic memory, speed-attention, visuospatial ability, language, numeric reasoning) and whether the associations extend to other common attributions for discrimination (age, gender, race).

Method-Participants $(N=2,593)$ were from the Harmonized Cognitive Assessment Protocol (HCAP) sub-study of the Health and Retirement Study (HRS). HCAP participants completed a battery of cognitive tasks that measured the five focal cognitive domains. Participants reported on their perceived experiences with discrimination at the previous regular HRS assessment.

Results-In models that accounted for demographic covariates and BMI, weight discrimination (reported by $6 \%$ of participants) was associated with a two-fold increased risk of poor performance on tasks of episodic memory, speed-attention, visuospatial ability, and numeric reasoning. Body mass index was largely unrelated to performance in the five cognitive domains. The other attributions for discrimination were generally unrelated to cognition, but there were sexand race-specific associations for gender and race discrimination, respectively.

Conclusions-The present study identified attribution- and domain-specific associations between discrimination and cognitive performance in older adulthood.

\footnotetext{
"Corresponding author at: Angelina R. Sutin, Ph.D., Florida State University College of Medicine, 1115 W. Call Street, Tallahassee. angelina.sutin@med.fsu.edu.

Publisher's Disclaimer: This is a PDF file of an unedited manuscript that has been accepted for publication. As a service to our customers we are providing this early version of the manuscript. The manuscript will undergo copyediting, typesetting, and review of the resulting proof before it is published in its final citable form. Please note that during the production process errors may be discovered which could affect the content, and all legal disclaimers that apply to the journal pertain.

Conflict of Interest

All authors have completed the Unified Competing Interest form. The authors have no competing interests to report.
} 


\section{Keywords}

Weight stigma; Neuropsychological tests; Cognitive aging; Psychosocial Stressor

Individuals with overweight and obesity are often the target of unfair treatment. ${ }^{1}$ Such weight stigma is common across multiple settings, including at work, with healthcare providers, and by family members or strangers out in public. ${ }^{2}$ In addition to being unpleasant in the moment, these experiences have widespread implications for mental and physical health (for a review, $\mathrm{see}^{3}$ ). Weight discrimination, for example, has been associated with poor mental health, ${ }^{4}$ physiological dysregulation, ${ }^{5}, 6$ chronic disease burden, ${ }^{7}$ and, ultimately, premature mortality. ${ }^{8}$

There is some evidence that the poor health outcomes associated with weight discrimination extend to cognitive outcomes: Individuals who perceive weight discrimination are at greater risk of incident dementia. ${ }^{9}$ Of note, this association is independent of body mass index (BMI), which indicates that the stigma associated with weight, rather than higher weight itself, increases risk of poor cognitive outcomes. There is also evidence, however, that weight discrimination is unrelated to performance in some specific cognitive domains. ${ }^{10}$ As such, it is unclear whether the detrimental association of weight discrimination on cognition is limited to severe impairments in overall function or whether it is associated with worse performance in specific cognitive domains not yet tested.

There are a number of factors that may contribute to the association between weight discrimination and cognitive performance. ${ }^{11}$ Individuals who perceive weight discrimination, for example, carry a higher burden of disease, ${ }^{10}$ are more likely to use substances like alcohol and tobacco, ${ }^{12}$ get lower quality sleep, ${ }^{13}$ and are less likely to engage in physical activity. ${ }^{14}$ Disease burden, ${ }^{15}$ substance use, ${ }^{16}$ sleep, ${ }^{17}$ and physical activity ${ }^{18}$ are associated with cognitive function and risk of cognitive impairment. Further, discrimination may erode self-efficacy, ${ }^{19}$ and reduced self-efficacy (in the form of perceived constraints on personal control) is associated with worse cognition..$^{20}$ Lower cognitive function, in turn, may be a risk factor for severe cognitive impairment late in life and thus may serve as one mechanism on the pathway from discrimination to dementia risk.

Other forms of discrimination have been associated with cognitive function. Discrimination based on race, for example, has been associated with worse episodic memory ${ }^{10,21}$ and processing speed. ${ }^{21}$ Further, previous research has found that African Americans who perceive discrimination had lower overall cognitive function and worse episodic memory and processing speed but no differences in semantic memory, working memory, or visuospatial ability. ${ }^{22}$ Although some studies have found that perceived discrimination, regardless of the reason, to be associated with worse memory, ${ }^{23}$ not all find this relation. ${ }^{24}$ Less research has addressed the relation between other forms of discrimination and cognitive function, but there is some evidence that discrimination based on sex and age is associated with better overall mental status. ${ }^{10}$ Few studies, except for Barnes and colleagues ${ }^{22}$ have examined the association between discrimination and cognitive outcomes other than impairment and memory. Unfair treatment may have differential associations across cognitive domains. For example, domains such as speed-attention may be more sensitive to 
psychosocial stressors. ${ }^{25,26}$ Speed-attention may be the most vulnerable to psychosocial stress because these functions are among the most basic cognitive functions. ${ }^{27}$ As such, there may be less ability to compensate and/or develop strategies to offset deficits.

The present research takes a relatively comprehensive approach to examine the association between weight discrimination and multiple domains of cognitive function and whether the pattern of associations between weight discrimination and cognition extends to other common attributions for discrimination. Specifically, we examine the association between perceived discrimination based on weight, age, gender, and race and performance in five domains of cognitive function: episodic memory, speed-attention, language, visuospatial skills, and numeric reasoning. In addition, across all attributions, we examine whether the associations vary by the target of the attribution (i.e., BMI for weight discrimination, age for age discrimination, sex for gender discrimination, and race and ethnicity for race discrimination). Finally, as a point of comparison for weight discrimination, we also report the association between BMI and cognitive function.

\section{Method}

\section{Participants and Procedure}

A subsample of participants from the Health and Retirement Study (HRS) were selected to participate in a comprehensive assessment of cognition. HRS is a study of adults living in America aged 50 years or older and their spouses. Participants who completed the regular 2016 HRS assessment and who were 65 years or older were eligible to participate in the Harmonized Cognitive Assessment Protocol (HCAP). A random subsample of eligible participants $(N=5,500)$ was invited to participate, and a total of 3,496 HRS participants completed at least some of the assessment. Detailed information on the HCAP assessment, sampling, and how to obtain the data can be found at https://hrs.isr.umich.edu/news/2016harmonized-cognitive-assessment-protocol-hcap-early-version-10. For the current research, participants from HCAP who also reported on their experiences with discrimination in the 2014 or 2016 Leave-Behind Questionnaire $(N=2,593)$ were included in the analysis. Participants who completed at least some of the HCAP assessment but who did not have information available on discrimination $(n=903)$ were older $(d=.18, \mathrm{p}<.01)$, more likely to be a race other than white $\left(\chi^{2}=51.44, p<.01\right)$, more likely to be of Latinx ethnicity $\left(\chi^{2}=38.38\right.$, $p<.01)$, and had fewer years of education $(d=.28, p<.01)$ than our analytic sample. There were no differences by participant sex.

\section{Measures}

Perceived discrimination.-Participants completed a 6-item version of the Everyday Experiences with Discrimination measure ${ }^{28}$ included in the Leave-Behind Questionnaire. After rating the items, participants were asked to attribute the unfair treatment (if reported) to a number of personal attributes, including weight, age, gender, and race. All attributions were assessed on a yes/no scale. ${ }^{29}$

Cognitive function.-The HCAP measure included tasks that tapped into five domains of cognitive function. See Weir and colleagues ${ }^{30}$ for detailed information on the cognitive 
tasks, protocol, and scoring. Episodic memory was assessed with the CERAD Word List Learning and Recall Task, the Brave Man, and the Wechsler Memory Scale Logical Memory I. Speed-attention was measured with Backward Count, the Letter Cancellation Test, Trails A and B, and the Symbol-Digit Modalities Test. Visuospatial skills were measured with CERAD Constructional Praxis and Raven's matrices. Language was measured with an animal fluency task. Numeric Reasoning was measured with the HRS Number Series. All measures were scored based on standard criteria ${ }^{30}$ and aggregated within domain for domains measured with multiple tasks. For the aggregation, the individual tasks within each domain were standardized to a mean of 0 and standard deviation of 1 and the mean taken across the measures. Trails A and B were multiplied by -1 before standardization so that the scoring would be in the same direction as the other measures in the speed-attention domain. In addition to the continuous scores, threshold scores were calculated as one standard deviation below the mean (coded as 1) compared to all other scores (coded as 0 ).

Covariates.-Sociodemographic covariates were self-reported age in years, sex (female $=1$, male $=0$ ), race (African American=1 [dummy variable 1], other/unknown=1 [dummy variable 2] both compared to white $=0$ ], Latinx ethnicity ( $y e s=1, \mathrm{no}=0$ ), and education in years. Body mass index (BMI) was derived as $\mathrm{kg} / \mathrm{m}^{2}$ from staff-measured weight and height at the 2014-2016 assessment.

Participants reported on other health-related factors in the same HRS wave as perceived discrimination (i.e., 2014 or 2016). Disease burden was the sum of seven health conditions (hypertension, diabetes, heart disease, cancer, lung disease, stroke, and arthritis). Alcohol consumption was the average number of alcoholic drinks consumed per day over the past three months. Physical activity was the mean of two items about the frequency of engagement in moderate and vigorous physical activity rated from 1 (more than once a week) to 4 (hardly ever or never). Smoking was the average number of cigarettes smoked per day. Sleep quality was the mean of four items that measured frequency of trouble falling asleep, waking during the night, waking too early, and feeling unrested in the morning rated from 1 (rarely or never) to 3 (most of the time). Perceived constraints on personal control were measured with the five items from the Sense of Control Scale ${ }^{31}$ (e.g., "I often feel helpless in dealing with the problems of life") on a scale from 1 (strongly disagree) to 6 (strongly agree). All potential mechanisms were scored in the direction of greater risk (e.g., lack of physical activity, worse sleep quality, greater constraints, etc.). In some analyses, these factors were included as additional covariates.

\section{Statistical Approach}

Linear regression was used to examine the association between the attributions for discrimination and the continuous cognitive domain scores, controlling for the sociodemographic covariates. The attributions were entered simultaneously (results were similar if entered separately). Logistic regression was used to examine whether the attributions (entered simultaneously) were associated with increased risk of performing at least one standard deviation below the mean for each domain. We also tested whether the association between the attributions for discrimination and the cognitive outcomes was moderated by the target of the attribution for discrimination. As such, we tested interactions 
between BMI and weight discrimination, age and age discrimination, sex and gender discrimination, and race/ethnicity and race discrimination. We did three additional sets of analyses. First, because weight discrimination is more prevalent among individuals with overweight or obesity than normal weight, ${ }^{32}$ we repeated the analyses limiting the sample to participants with a BMI $\geq 25$. Second, for comparison, we report the associations between BMI and the five cognitive domains. Specifically, we used linear regression to test the association between BMI and the cognitive domain scores, logistic regression to test the association with risk of performing at least one standard deviation below the mean in each domain, and multivariate analysis of covariance (MANCOVA) to test whether cognitive performance differed by BMI category, comparing the overweight (BMI 25-29.9) and obesity (BMI 230 ) categories to normal weight (BMI between 18.50 and 24.99). All analyses for BMI controlled for the covariates. Third, we tested the associations with weight discrimination further adjusting for the additional potential confounders/mediators.

\section{Results}

Table 1 shows the descriptive statistics for all study variables. The sample included $60 \%$ women, the average age was over 75, and the average BMI was almost 30 (scores $\geq 30$ meet criteria for obesity). Discrimination based on age was the most prevalent (31\%), followed by discrimination based on gender (10\%), race (7\%), and weight (6\%). Table 2 shows the results of the linear and logistic regressions. Weight discrimination was associated with significantly worse performance on tasks that measured speed-attention, visuospatial abilities, and numeric reasoning. Weight discrimination was further associated with an approximately 2-fold increased risk of scoring at least one standard deviation below the mean on memory, as well as speed-attention, visuospatial abilities, and numeric reasoning (Table 2). None of the associations between weight discrimination and the cognitive domains was moderated by BMI (Table 3). The linear associations were similar when the sample was limited to participants with overweight or obesity $\left(\beta_{\text {Memory }}=-.02, n s, n=2,016\right.$; $\beta_{\text {Speed-attention }}=-.04, p<.05, n=2,015 ; \beta_{V_{\text {isuospatial abilities }}=-.07, p<.01, n=2,012 ; \beta_{\text {Language }}=}=$ $\left.-.01, n s, n=2,016 ; \beta_{\text {Numeric reasoning }}=-.04, n s, n=1,781\right)$. And, likewise, the results of the logistic regressions were similar when the sample was limited to participants with overweight or obesity $\left(\mathrm{OR}_{\text {Memory }}=2.35,95 \% \mathrm{CI}=1.37-4.02, n=2,016 ; \mathrm{OR}_{\text {Speed-attention }}=1.84\right.$, 95\% CI=1.01-3.34, $n=2,015$; $\mathrm{OR}_{\text {Visuospatial abilities }}=1.89,95 \% \mathrm{CI}=1.14-3.14, n=2,012$; $\mathrm{OR}_{\text {Language }}=1.3195 \% \mathrm{CI}=.78-2.20, n=2,016 ; \mathrm{OR}_{\text {Numeric reasoning }}=1.96,95 \% \mathrm{CI}=1.10-3.47$, $n=1,781$ ). The pattern of associations was similar, but reduced, when disease burden, alcohol consumption, physical activity, smoking, sleep quality, and perceived constraints were added as additional covariates $\left(\beta_{\text {Memory }}=-.01, n s, n=2,536 ; \beta_{\text {Speed-attention }}=-.03, n s, n=2,532\right.$; $\beta_{\text {Visuospatial abilities }}=-.05, p<.01, n=2,532 ; \beta_{\text {Language }}=.00, n s, n=2,536 ; \beta_{\text {Numeric reasoning }}=$ $-.03, n s, n=2,249)$. These associations, however, should be interpreted with caution because some of the additional covariates could be considered as mediators as well as confounders.

By comparison, BMI was generally unrelated or associated with better cognitive function (Table 4). Higher BMI was associated with better memory function in both the linear and logistic analyses, better performance in the speed-attention domain in the logistic analysis, and individuals with overweight performed better in this domain than individuals of normal 
weight. Finally, higher BMI was associated with worse performance on the numeric ability task in the continuous analysis, but not in the logistic analysis or the MANCOVA analysis.

Finally, across the other three attributions for discrimination, there was only one main effect $^{1}$ (Table 2): Participants who reported gender discrimination performed better on numeric reasoning. Sex did, however, moderate the relation between gender discrimination and four of the five cognitive domains (Table 3). Specifically, men who reported discrimination based on gender performed worse in the memory, speed-attention, visuospatial, and language domains, whereas these interactions indicated that women who reported discrimination based on gender performed slightly better in the memory and language domains and was unrelated to performance in the speed-attention and visuospatial domains. A somewhat similar pattern emerged for discrimination based on race. White participants who reported discrimination based on race performed worse in the speedattention, visuospatial, and numeracy domains, whereas race discrimination was unrelated to performance in these domains among African American participants. The association between race discrimination and the cognitive domains was not moderated by Latinx ethnicity. Age discrimination was not associated with the cognitive measures and none of the associations was moderated by age. In the logistic analysis, there was one association between race discrimination and better memory function (Table 2).

\section{Discussion}

The present study indicated that unfair treatment on the basis of weight was associated with worse performance in specific cognitive domains. In particular, weight discrimination was associated with an about two-fold increased risk of poor performance on tasks that measured episodic memory, speed-attention, visuospatial ability, and numeric reasoning. By comparison, body mass index was generally unrelated to performance across the five cognitive domains. At the sample level, these associations did not extend to other common attributions for discrimination. There was, however, evidence of interactions between the target characteristic and gender and race discrimination on cognitive function.

Previous research on discrimination and cognition has suggested that individuals who perceive unfair treatment tend to perform worse on tasks of cognitive function, ${ }^{22}$ although not all find this relation. ${ }^{24}$ The relation between weight discrimination and cognition has likewise been mixed. Weight discrimination in the HRS, for example, has been associated with risk of significant impairment ${ }^{9}$ but unrelated to memory function. ${ }^{10}$ The present research starts to reconcile these disparate findings and suggests that there is a threshold effect for weight discrimination on memory. That is, there does not seem to be a linear association between weight discrimination and memory performance; rather, individuals who experience weight discrimination are more likely to perform really poorly on memory tasks, thus the association past a threshold. Also consistent with previous work on discrimination and cognition, ${ }^{22}$ weight discrimination was associated with worse

\footnotetext{
${ }^{1}$ The sum of the four attributions was also largely unrelated to cognitive function (median $\beta=-.02$ ), which is likely because the associations for age, gender, and race discrimination were generally either zero or positive, so combining them with weight discrimination into one measure essentially eliminated the associations; only the association with visuospatial abilities $(\beta=-.03, p=.04)$ was statistically significant.
} 
performance in the speed-attention domain. This association may be due, in part, to the social stress of weight discrimination, since stress can impair processing speed. ${ }^{25}$

There was little evidence that the other common attributions for discrimination were associated with cognitive function in the specific domains. A noteworthy pattern, however, emerged from the interaction analyses for gender and race discrimination. Specifically, the association between discrimination and cognitive performance was worse in groups that are not the historical target of the attribution for discrimination (i.e., men and white participants) whereas performance was either better or unrelated to discrimination in the groups who are the historical targets (i.e., women and African Americans). This pattern is similar to what we found previously using different cognitive measures in the HRS. ${ }^{10}$ Women and African Americans with higher education and higher cognitive function may be more likely to be in situations, such as the workforce, where they may experience discrimination. And, indeed, African Americans are more likely to report discrimination at higher incomes and education, whereas white individuals are more likely to report it at lower incomes and education. ${ }^{33}$ Interestingly, there was no association between age discrimination and cognition, which is inconsistent with the literature on negative self-perceptions of aging and worse cognitive outcomes. ${ }^{34}$ It may be that internalized views of aging are more important for cognitive performance ${ }^{35}$ than perceived unfair treatment on the basis of age.

The inclusion of the additional covariates in the analysis reduced the association between weight discrimination and cognitive function. This reduction may be due to either residual confounding or mechanisms of the association between weight discrimination and cognitive function. For example, disease burden may be a consequence of obesity that contributes to cognitive outcomes rather than a consequence of weight discrimination. There is evidence, however, that individuals who perceive weight discrimination tend to increase in disease burden over time, ${ }^{10}$ which may be a risk factor for incident dementia. ${ }^{15,20}$ With the current data, however, it is not possible to disentangle mediational pathways from confounding variables. As such, these associations should be interpreted with caution due to the possibility of over adjustment.

The stress associated with weightism may be a factor that contributes to worse cognitive performance. Stress, for example, has physiological effects on the body that are also associated with cognition. Stress is associated with greater systemic inflammation and cortisol, ${ }^{36}$ which is also implicated in worse cognitive performance over time. ${ }^{37,}{ }^{38} \mathrm{In}$ addition, stress may promote rumination and other psychological interference ${ }^{39}$ that reduce the ability to perform well on tests of cognitive function. ${ }^{40}$ And, indeed, weight discrimination is associated with greater systemic inflammation ${ }^{41}$ and cortisol reactivity, ${ }^{42}$ and greater rumination. ${ }^{43}$ It is likely that multiple pathways contribute to the association between weight discrimination and cognitive function.

The pattern of associations in the current study does raise the question, however, as to why the associations would be apparent for weight discrimination but not for other forms of discrimination that are presumably as stressful. There are several potential reasons. First, the perpetrators of weight discrimination are often family members or close friends, in addition to strangers. ${ }^{44}$ As such, the people who should be a source of support and safety for the 
individual may be harmful. Second, because weight discrimination continues to remain socially acceptable, people who experience weight discrimination may get less support and understanding from their social support networks than individuals who experience discrimination based on other personal characteristics. That is, although the other forms of discrimination were more prevalent than weight discrimination (e.g., $31 \%$ for age discrimination versus $6 \%$ for weight discrimination), there may be less support offered by close others than for the other forms of discrimination. For example, a spouse may express sympathy for being mistreated because of age but be less sympathetic for mistreatment because of weight. Third, there may be differences based on the perceived controllability of the characteristics. That is, individuals who experience weight discrimination may be more likely to blame themselves because they hold the belief that they have control over their body weight and/or have internalized negative attitudes toward higher weight. ${ }^{45}$ In contrast, individuals tend to have less control over their sex, race, and age. The greater shame and internalization of discrimination based on weight may take a greater toll of the cognitive health of the individual.

The present research had several strengths, including a relatively large sample of older adults, a comprehensive assessment of cognitive function, and multiple attributions for discrimination. There are also limitations. For example, there was no information available about the source, setting, or frequency of each attribution for the unfair treatment, and the cognitive data were assessed only once. In future research, it would be helpful to have more information about the experience of each form of discrimination and how perceived discrimination is associated with change in the five cognitive domains over time. Further, more work needs to address and identify the distinct mechanisms that explain why weight discrimination, but not other forms of discrimination, is associated with worse cognitive performance. Future work is also needed to distinguish between mediation and confounding for intervening variables. Despite these limitations, the present research provides evidence that unfair treatment on the basis of body weight is associated with intermediate markers of cognitive health and suggests a pathway to impairment.

\section{Acknowledgement}

This work was supported by the National Institute on Aging of the National Institutes of Health under Award Number R01AG053297 and R21AG057917. The content is solely the responsibility of the authors and does not necessarily represent the official views of the National Institutes of Health. The Health and Retirement Study (HRS) is sponsored by the National Institute on Aging (NIAU01AG009740) and conducted by the University of Michigan. HRS was approved by the University of Michigan Institutional Review Board. HRS data are available at: http:// hrsonline.isr.umich.edu/index.php.

\section{References}

1. Puhl RM, Andreyeva T, Brownell KD. Perceptions of weight discrimination: prevalence and comparison to race and gender discrimination in America. Int J Obesity 2008; 32: 992-1000.

2. Puhl RM, Brownell KD. Confronting and coping with weight stigma: an investigation of overweight and obese adults. Obesity 2006; 14: 1802-15. [PubMed: 17062811]

3. Tomiyama AJ, Carr D, Granberg EM, Major B, Robinson E, Sutin AR et al. How and why weight stigma drives the obesity 'epidemic' and harms health. BMC Med 2018; 16: 123. [PubMed: 30107800] 
4. Robinson E, Sutin A, Daly M. Perceived weight discrimination mediates the prospective relation between obesity and depressive symptoms in U.S. and U.K. adults. Health Psychol 2017; 36: 112121. [PubMed: 27748611]

5. Vadiveloo M, Mattei J. Perceived weight discrimination and 10-year risk of allostatic load among US adults. Ann Behav Med 2017; 51: 91-104.

6. Daly M, Sutin AR, Robinson E. Perceived weight discrimination mediates the prospective association between obesity and physiological dysregulation: Evidence from a population-based cohort. Psychol Sci 2019; 30: 1030-1039. [PubMed: 31158067]

7. Udo T, Purcell K, Grilo CM. Perceived weight discrimination and chronic medical conditions in adults with overweight and obesity. Int J Clin Pract 2016; 70: 1003-1011. [PubMed: 28032427]

8. Sutin AR, Stephan Y, Terracciano A. Weight discrimination and risk of mortality. Psychol Sci 2015; 26: 1803-1811. [PubMed: 26420442]

9. Sutin AR, Stephan Y, Robinson E, Daly M, Terracciano A. Perceived weight discrimination and risk of incident dementia. Int J Obes 2019; 43: 1130-1134.

10. Sutin AR, Stephan Y, Carretta H, Terracciano A. Perceived discrimination and physical, cognitive, and emotional health in older adulthood. Am J Geriatr Psychiatry 2015; 23: 171-179. [PubMed: 24745563]

11. Pascoe EA, Smart Richman L. Perceived discrimination and health: A meta-analytic review. Psychol Bull 2009; 135: 531-54. [PubMed: 19586161]

12. Sutin AR, Terracciano A. Perceived weight discrimination and high-risk health-related behaviors. Obesity 2017; 25: 1183-1186. [PubMed: 28653501]

13. Vaghela P, Sutin AR. Discrimination and sleep quality among older US adults: the mediating role of psychological distress. Sleep Health 2016; 2: 100-108. [PubMed: 28923251]

14. Phibbs $S$, Thorburn S, Branscum AJ. Is weight discrimination associated with physical activity among middle aged and older adults? J Prim Prev 2019; 40: 279-295. [PubMed: 30895424]

15. Kivipelto M, Ngandu T, Fratiglioni L, Viitanen M, Kåreholt I, Winblad B et al. Obesity and vascular risk factors at midlife and the risk of dementia and Alzheimer disease. Arch Neurol 2005; 62: 1556-60. [PubMed: 16216938]

16. Norton S, Matthews FE, Barnes DE, Yaffe K, Brayne C. Potential for primary prevention of Alzheimer's disease: An analysis of population-based data. Lancet Neurol 2014; 13: 788-94. [PubMed: 25030513]

17. Dzierzewski JM, Dautovich N, Ravyts S. Sleep and cognition in older adults. Sleep Med Clin 2018; 13: 93-106. [PubMed: 29412987]

18. Prakash RS, Voss MW, Erickson KI, Kramer AF. Physical activity and cognitive vitality. Annu Rev Psychol 2015; 66: 769-97. [PubMed: 25251492]

19. Major B, Hunger JM, Bunyan DP, Miller CT. The ironic effects of weight stigma. J Exp Soc Psychol 2014; 51: 74-80.

20. Sutin AR, Stephan Y, Terracciano A. Psychological distress, self-beliefs, and risk of cognitive impairment and dementia. J Alzheimers Dis 2018; 65: 1041-1050. [PubMed: 30103318]

21. Thames AD, Hinkin CH, Byrd DA, Bilder RM, Duff KJ, Mindt MR et al. Effects of stereotype threat, perceived discrimination, and examiner race on neuropsychological performance: simple as black and white? J Int Neuropsychol Soc 2013; 19: 583-93. [PubMed: 23388089]

22. Barnes LL, Lewis TT, Begeny CT, Yu L, Bennett DA, Wilson RS. Perceived discrimination and cognition in older African Americans. J Int Neuropsychol Soc 2012; 18: 856-65. [PubMed: 22595035]

23. Shankar A, Hinds P. Perceived discrimination: Associations with physical and cognitive function in older adults. Health Psychol 2017; 36: 1126-1134. [PubMed: 28639821]

24. Zahodne LB, Manly JJ, Smith J, Seeman T, Lachman ME. Socioeconomic, health, and psychosocial mediators of racial disparities in cognition in early, middle, and late adulthood. Psychol Aging 2017; 32: 118-130. [PubMed: 28287782]

25. Korten NC, Comijs HC, Penninx BW, Deeg DJ. Perceived stress and cognitive function in older adults: which aspect of perceived stress is important? Int J Geriatr Psychiatry 2017; 32: 439-445. [PubMed: 27059116] 
26. Allen AP, Curran EA, Duggan Á, Cryan JF, Chorcoráin AN, Dinan TG et al. A systematic review of the psychobiological burden of informal caregiving for patients with dementia: Focus on cognitive and biological markers of chronic stress. Neurosci Biobehav Rev 2017; 73: 123-164. [PubMed: 27986469]

27. Lezak MD. Neuropsychological assessment, 4th edn Oxford University Press: New York, 2004.

28. Williams DR, Yu Y, Jackson JS, Anderson NB. Racial differences in physical and mental health. Socio-economic status, stress and discrimination. J Health Psychol 1997; 2: 335-351. [PubMed: 22013026]

29. Kessler RC, Mickelson KD, Williams DR. The prevalence, distribution, and mental health correlates of perceived discrimination in the United States. J Health Soc Beh 1999; 40: 208-230.

30. Weir DR, Langa KM, Ryan LH. Harmonized Cognitive Assessment Protocol (HCAP): Study Protocol Summary, 2016.

31. Lachman ME, Weaver SL. The sense of control as a moderator of social class differences in health and well-being. J Pers Soc Psychol 1998; 74: 763-73. [PubMed: 9523418]

32. Dutton GR, Lewis TT, Durant N, Halanych J, Kiefe CI, Sidney S et al. Perceived weight discrimination in the CARDIA study: differences by race, sex, and weight status. Obesity 2014; 22: 530-6. [PubMed: 23512948]

33. Mayrl D, Saperstein A. When white people report racial discrimination: The role of region, religion, and politics. Soc Sci Res 2013; 42: 742-54. [PubMed: 23521992]

34. Robertson DA, King-Kallimanis BL, Kenny RA. Negative perceptions of aging predict longitudinal decline in cognitive function. Psychol Aging 2016; 31: 71-81. [PubMed: 26691302]

35. Stephan Y, Sutin AR, Luchetti M, Terracciano A. Feeling Older and the Development of Cognitive Impairment and Dementia. J Gerontol B Psychol Sci Soc Sci 2017; 72: 966-973. [PubMed: 27436103]

36. Rohleder N Stimulation of systemic low-grade inflammation by psychosocial stress. Psychosom Med 2014; 76: 181-9. [PubMed: 24608036]

37. Teunissen CE, van Boxtel MP, Bosma H, Bosmans E, Delanghe J, De Bruijn C et al. Inflammation markers in relation to cognition in a healthy aging population. J Neuroimmunol 2003; 134: 142 50. [PubMed: 12507782]

38. Staufenbiel SM, Penninx BW, Spijker AT, Elzinga BM, van Rossum EF. Hair cortisol, stress exposure, and mental health in humans: a systematic review. Psychoneuroendocrinology 2013; 38: 1220-35. [PubMed: 23253896]

39. Brosschot JF, Gerin W, Thayer JF. The perseverative cognition hypothesis: a review of worry, prolonged stress-related physiological activation, and health. J Psychosom Res 2006; 60: 113-24. [PubMed: 16439263]

40. Munoz E, Sliwinski MJ, Smyth JM, Almeida DM, King HA. Intrusive thoughts mediate the association between neuroticism and cognitive function. Pers Individ Dif 2013; 55: 898-903. [PubMed: 24683284]

41. Sutin AR, Stephan Y, Luchetti M, Terracciano A. Perceived weight discrimination and C-reactive protein. Obesity 2014; 22: 1959-61. [PubMed: 24828961]

42. Himmelstein MS, Incollingo Belsky AC, Tomiyama AJ. The weight of stigma: cortisol reactivity to manipulated weight stigma. Obesity 2015; 23: 368-74. [PubMed: 25522347]

43. Wang SB, Lydecker JA, Grilo CM. Rumination in patients with binge-eating disorder and obesity: Associations with eating-disorder psychopathology and weight-bias internalization. Eur Eat Disord Rev 2017; 25: 98-103. [PubMed: 28078784]

44. Sutin AR, Terracciano A. Sources of weight discrimination and health. Stigma Health 2017; 2: $23-$ 27. [PubMed: 28289702]

45. Pearl RL, Lebowitz MS. Beyond personal responsibility: Effects of causal attributions for overweight and obesity on weight-related beliefs, stigma, and policy support. Psychol Health 2014; 29: 1176-1191. [PubMed: 24754230] 


\section{Highlights:}

- Weight discrimination was associated with worse cognitive function in four domains

- The associations were independent of measured body mass index

- Gender and race discrimination had counterintuitive associations with cognition

- Age discrimination was unrelated to cognitive function 


\section{롤 \\ 일}

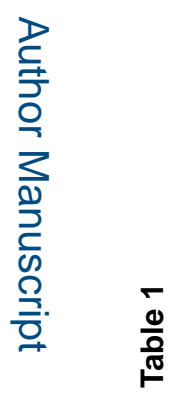

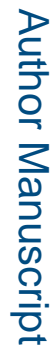

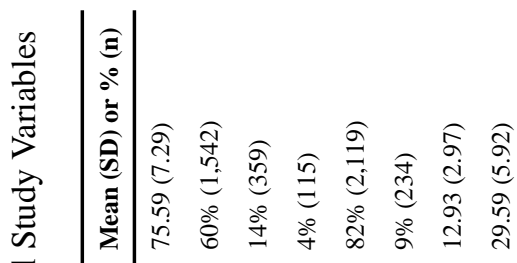

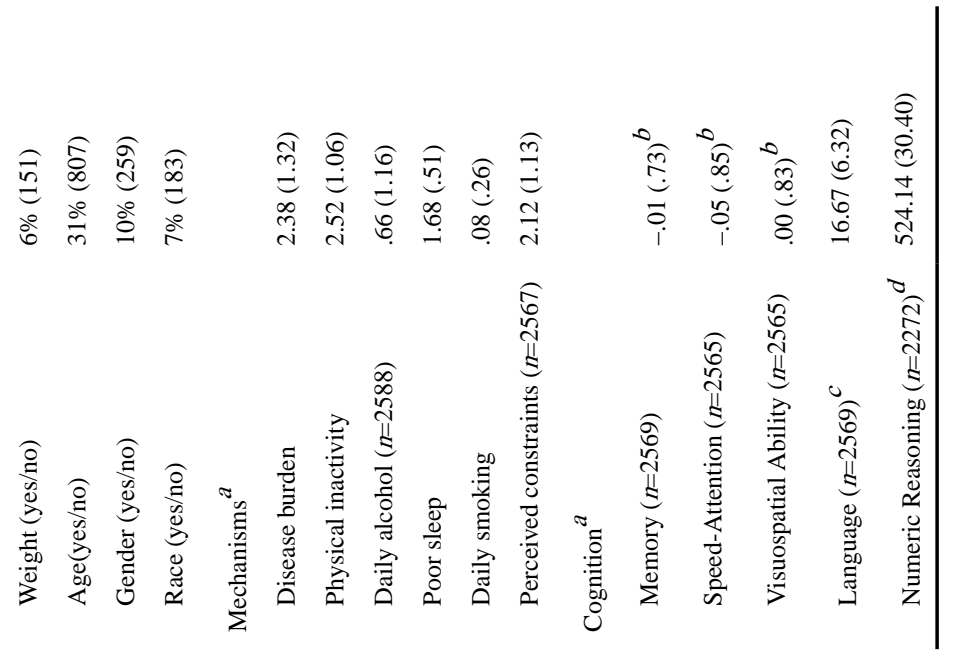

론

J Psychosom Res. Author manuscript; available in PMC 2021 April 01. 


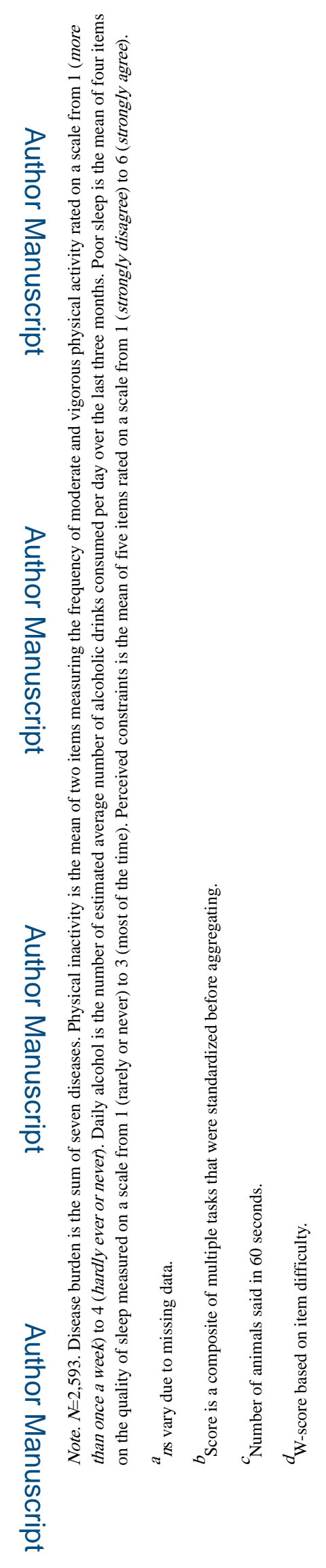

J Psychosom Res. Author manuscript; available in PMC 2021 April 01. 


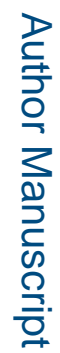

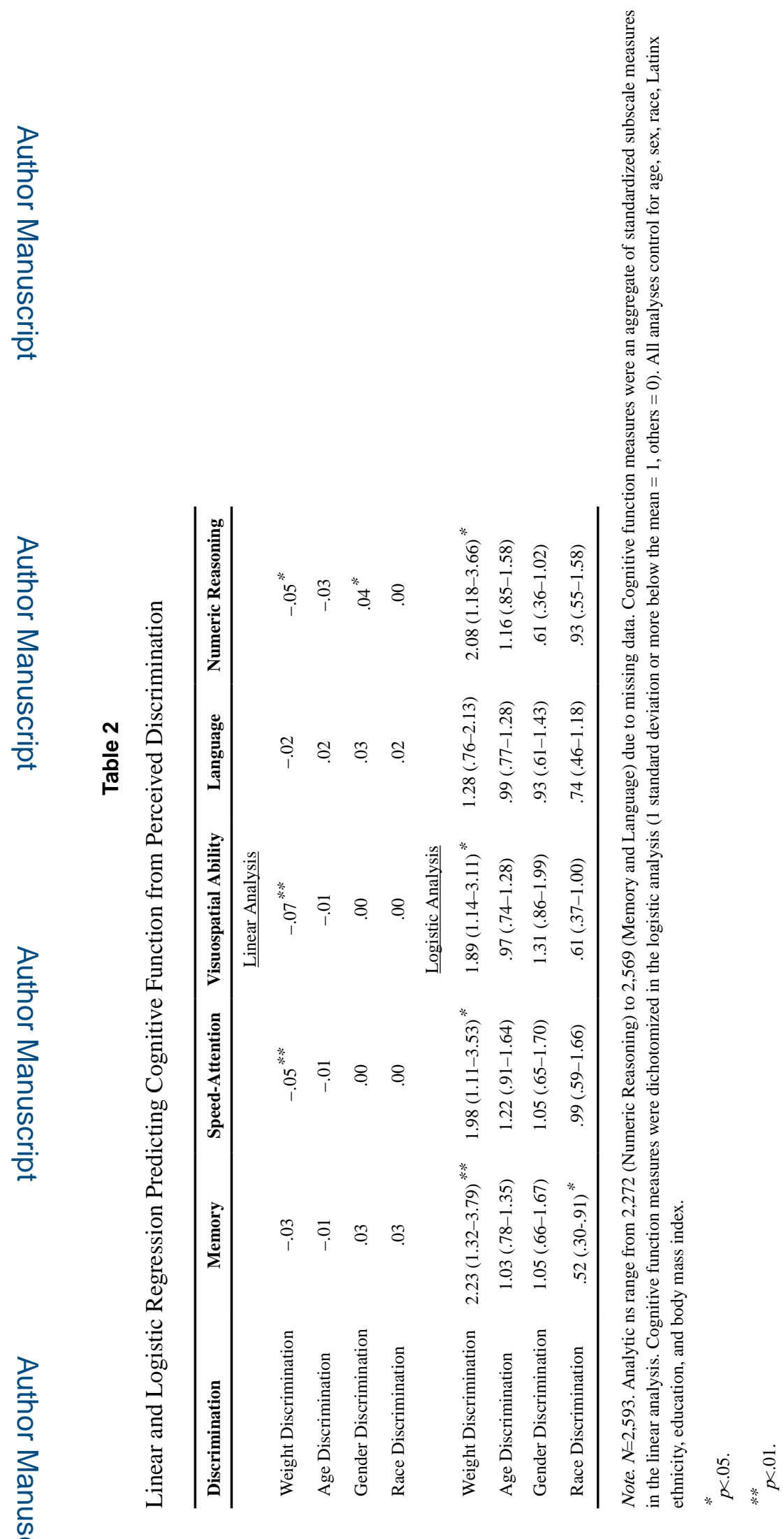

J Psychosom Res. Author manuscript; available in PMC 2021 April 01. 


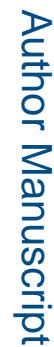

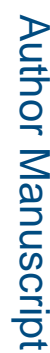

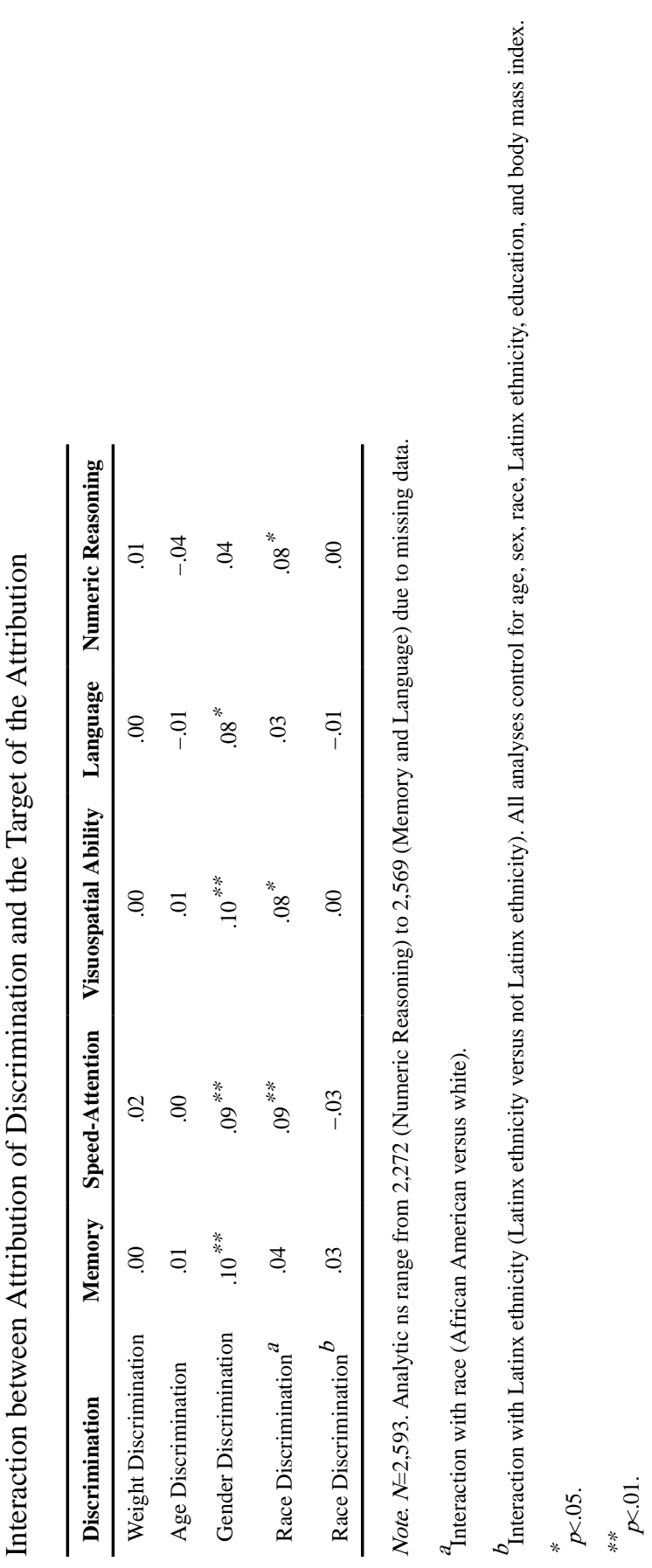

J Psychosom Res. Author manuscript; available in PMC 2021 April 01. 


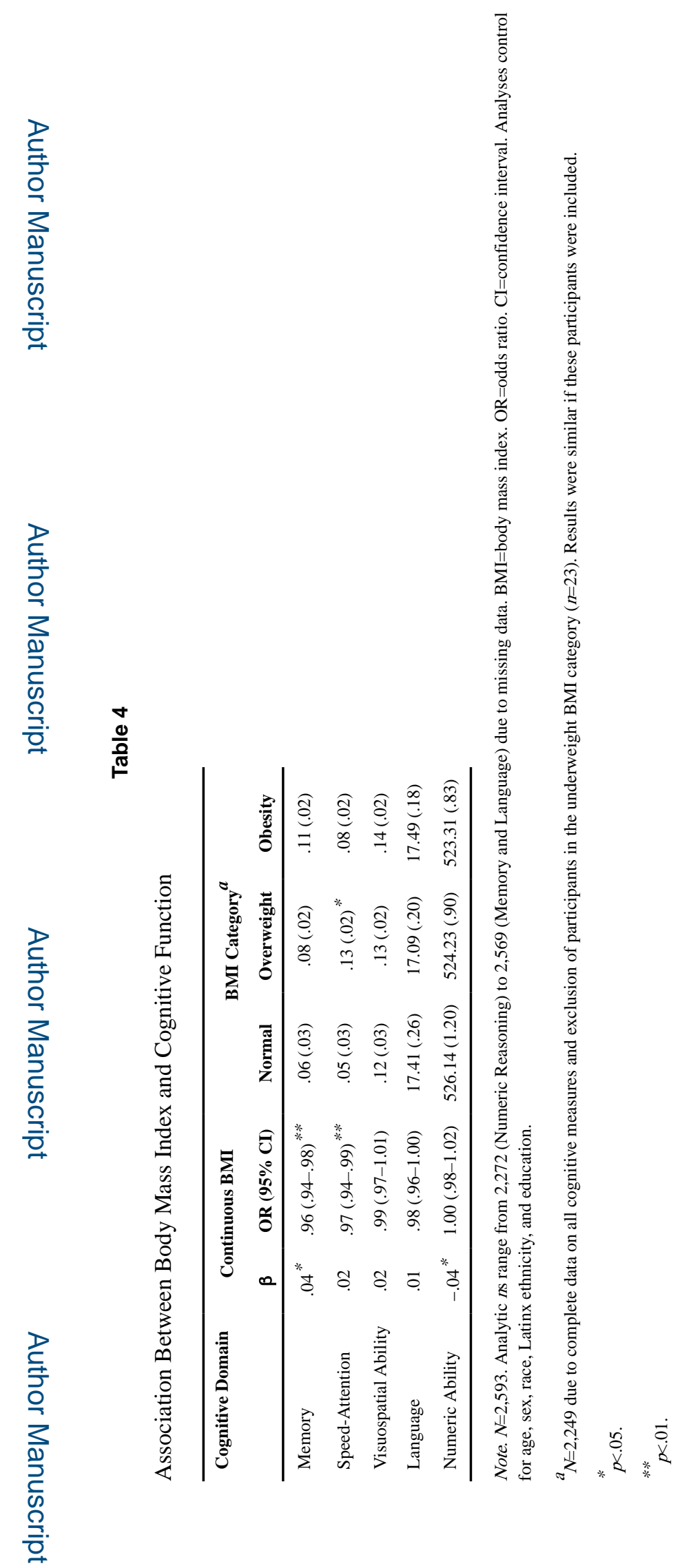

J Psychosom Res. Author manuscript; available in PMC 2021 April 01. 